\title{
Correction to: Left ventricular dyssynchrony and abnormalities in wall motion, assessed by gated-SPECT as ischemic auxiliary markers
}

Correction to: Journal of Nuclear Cardiology (2018)

https://doi.org/10.1007/s12350-018-01544-4

1. In Figure 3, sensitivity and specificity were interchanged. The corrected Figure 3 is shown below.

2. The author names listed in reference 14 have been modified; the correct reference reads: Nakanishi R, Gransar H, Slomka P, Arsanjani R, Shalev A, Otaki
$\mathrm{Y}$, et al. Predictors of high-risk coronary artery disease in subjects with normal SPECT myocardial perfusion imaging. J Nucl Cardiol 2016;23:530-41.

3. The units of standard deviation (SD) and bandwidth (BW) in the abstract, results and in Table 3 are expressed in degrees from 0 to $360^{\circ}$.

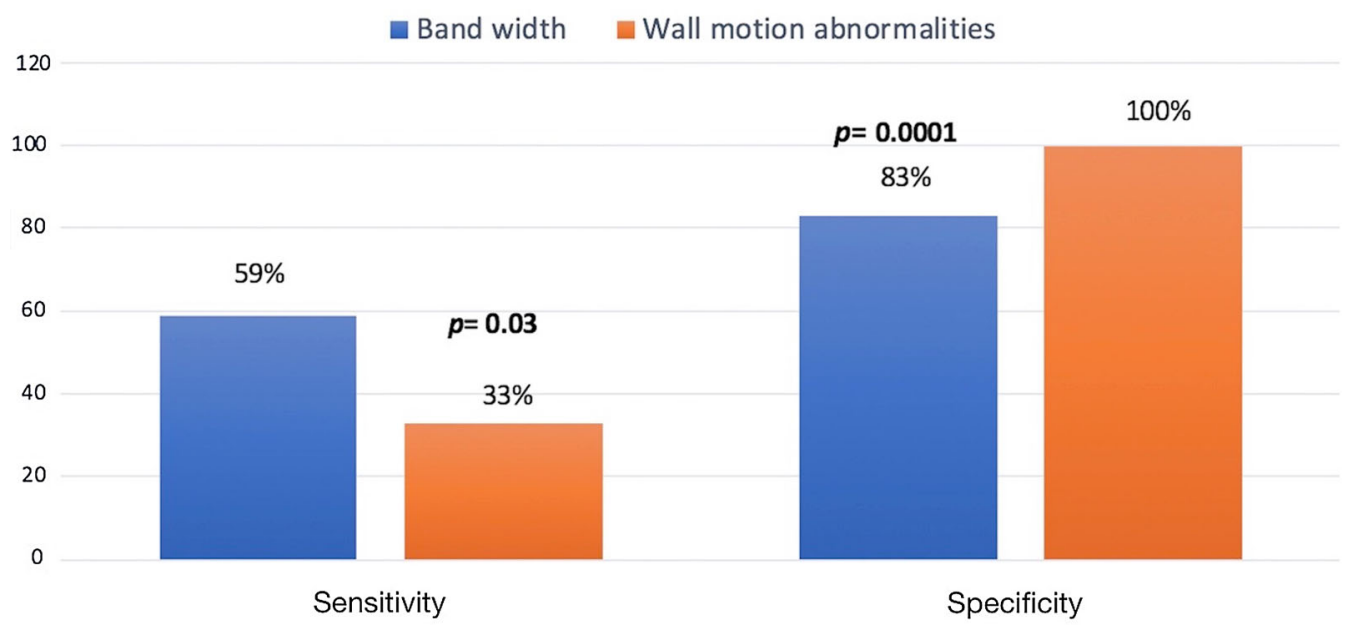

Figure 3. Analysis of sensitivity and specificity for detection of myocardial ischemia, left ventricular dyssynchrony, and alterations in regional wall motion. 